\title{
PREDICTING INTEGRALS OF RANDOM FIELDS USING OBSERVATIONS ON A LATTICE ${ }^{1}$
}

\author{
By Michael L. Stein \\ University of Chicago
}

\begin{abstract}
For a stationary random field $Z$ on $\mathbb{R}^{d}$, this work studies the asymptotic behavior of predictors of $\int v(x) Z(x) d x$ based on observations on a lattice as the distance between neighbors in the lattice tends to 0 . Under a mild condition on the spectral density of $Z$, an asymptotic expression for the mean-squared error of a predictor of $\int v(x) Z(x) d x$ based on observations on an infinite lattice is derived. For predicting integrals over the unit cube, a simple predictor based just on observations in the unit cube is shown to be asymptotically optimal if $v$ is sufficiently smooth and $Z$ is not too smooth. Modified predictors extend this result to smoother processes.
\end{abstract}

1. Introduction. This work studies the behavior of linear predictors of integrals of stationary random fields based on observations on a regular lattice. Ripley [(1981), Chapter 3], Tubilla (1975), Matern [(1986), Chapter 5], Cressie [(1993), page 321] and Stein (1993), among others, consider the asymptotic properties of predictors of area averages of random fields, that is, predicting $\int_{B} Z(x) d x$ over some set $B$, often a square, for a random field $Z: \mathbb{R}^{d} \rightarrow \mathbb{R}$. Schoenfelder $(1978,1982)$ and Schoenfelder and Cambanis (1982) studied the more general problem of predicting $\int v(x) Z(x) d x$. However, none of these works investigates whether or not these predictors are asymptotically efficient relative to the optimal linear predictors. Under a broad class of models for $Z$, this paper derives an expression for the asymptotic variance of the optimal predictor of $\int v(x) Z(x) d x$ based on an infinite lattice of observations as the lattice spacing tends to 0 . In addition, explicit expressions are obtained for predictors of $\int_{[0,1]^{d}} v(x) Z(x) d x$ based just on observations in $[0,1]^{d}$ that achieve this asymptotic mean-squared error if $v$ is smooth and $Z$ is not too smooth. It follows that these predictors are asymptotically optimal when the conditions on $v$ and $Z$ are satisfied.

Section 2 studies the following scenario: for a stationary process $Z$ on $\mathbb{R}^{d}$, suppose we observe $Z(\delta(J+\nu))$ for all $J$ in $\mathbb{Z}^{d}$, the $d$-dimensional integer lattice, where $\delta$ is a positive scalar and $\nu$ a fixed vector in $\mathbb{R}^{d}$. Under condition (2.1) on the spectral density of $Z$, Section 2 derives an asymptotic

\footnotetext{
Received November 1991; revised May 1995.

${ }^{1}$ This research was supported in part by NSF Grants DMS-89-02267 and DMS-92-04504. This manuscript was prepared using computer facilities supported in part by NSF Grants DMS-8905292, DMS-87-03942, DMS-86-01732 and DMS-84-04941 awarded to the Department of Statistics at the University of Chicago, and by the University of Chicago Block Fund.

AMS 1991 subject classifications. Primary 62M40; secondary 62M20.

Key words and phrases. Spatial statistics, numerical integration, optimal prediction.
} 
expression for the mean-squared error of the predictor of $\int v(x) Z(x) d x$ as $\delta$, the distance between neighboring observations, tends to 0 .

Since, in practice, one never has observations on an infinite lattice, this result is of limited utility by itself. However, it does serve as a benchmark for predictors based on a finite lattice. If we can show that a predictor based on a finite lattice has the same asymptotic variance as the optimal predictor based on an infinite lattice, then it is obviously asymptotically efficient relative to the optimal predictor on the finite lattice. Section 3 considers predicting $\int_{[0,1]^{d}} v(x) Z(x) d x$ by

$$
\sum_{J} v\left(\frac{J-h}{m}\right) Z\left(\frac{J-h}{m}\right),
$$

where $h=\frac{1}{2}(1, \ldots, 1), m$ is a positive integer and each component of $J$ varies from 1 through $m$. If $v$ has bounded partial derivatives of order $d+1$ on $[0,1]^{d}$ and $Z$ is not too smooth, then Proposition 3.1 shows that this simple predictor is asymptotically optimal. The condition that $Z$ not be too smooth is that its spectral density $f(\omega)$ must decrease more slowly than $|\omega|^{-q}$ as $|\omega| \rightarrow \infty$ for some $q<4$. Since, under (2.1), $f(\omega)=o\left(|\omega|^{-d}\right)$ is necessary for $f$ to be a spectral density, Proposition 3.1 is only meaningful in at most three dimensions. Section 4 demonstrates how the results of Section 3 extend to larger $q$ and hence larger $d$ by modifying the predictor.

2. Asymptotic variance of optimal predictors. Suppose $Z$ is a weakly stationary random field on $\mathbb{R}^{d}$ with mean 0 and spectral density $f$. Throughout this paper, we will assume

$$
f(\omega) \asymp \gamma(|\omega|) \text { as }|\omega| \rightarrow \infty \text {, where } \gamma(t) \text { is regularly varying as } t \rightarrow \infty \text {. }
$$

See Feller [(1971), page 276] for the definition and properties of regularly varying functions. For positive functions $a$ and $b$ on $\mathbb{R}^{d}$, the notation $a(\omega) \asymp$ $b(\omega)$ means that $a(\omega) / b(\omega)$ is bounded away from 0 and $\infty ; a(\omega) \asymp b(\omega)$ as $|\omega| \rightarrow \infty$ means that, for some $R>0, a(\omega) / b(\omega)$ is bounded away from 0 and $\infty$ for all $|\omega| \geq R$. For $v: \mathbb{R}^{d} \rightarrow \mathbb{R}$ measurable and square integrable, define $V(\omega)=\int v(x) e^{i \omega^{\prime} x} d x$. Assume $\int|V(\omega)|^{2} f(\omega) d \omega<\infty$, which implies that $\int v(x) Z(x) d x$ is well defined as a mean-squared limit of finite weighted sums of $Z$ with $\operatorname{var}\left(\int v(x) Z(x) d x\right)=\int|V(\omega)|^{2} f(\omega) d \omega$.

Consider predicting $\int v(x) Z(x) d x$ based on observing $Z(\delta(J+\nu))$ for all $J \in \mathbb{Z}^{d}$, where $\delta>0$ and $\nu \in[0,1)^{d}$. Let $\hat{Z}_{\delta}(x)$ be the optimal linear predictor of $Z(x)$ based on the infinite lattice of observations, so that the optimal linear predictor of $\int v(x) Z(x) d x$ is $\int v(x) \hat{Z}_{\delta}(x) d x$. Finally, set

$$
S(t)=\int_{A(t)} \sum^{\prime} f(\omega+2 \pi t J)|V(\omega)|^{2} d \omega,
$$

where $A(t)=t(-\pi, \pi)^{d}$ and $\Sigma^{\prime}$ means summing over all elements of $\mathbb{Z}^{d}$ except the origin. 
Proposition 2.1. Under (2.1), if $f(\omega)>0$ almost everywhere with respect to Lebesgue measure, $\int v(x)^{2} d x<\infty$ and $\operatorname{var}\left(\int v(x) Z(x) d x\right)<\infty$, then $\operatorname{var}\left(\int v(x)\left\{Z(x)-\hat{Z}_{\delta}(x)\right\} d x\right) \sim S\left(\delta^{-1}\right)$ as $\delta \downarrow 0$.

Proof. The proof has two basic steps. First, show that there is a possibly suboptimal linear predictor that has asymptotic mean-squared error $S\left(\delta^{-1}\right)$. Second, show that the optimal predictor cannot have mean-squared error asymptotically smaller than $S\left(\delta^{-1}\right)$.

Let $U_{\delta, \nu}$ be the Hilbert space given by the real linear hull of the functions $e^{i \delta \omega^{\prime}(J+\nu)}$ for $J \in \mathbb{Z}^{d}$ with respect to the inner product $\left(\phi_{1}, \phi_{2}\right)=$ $\int \phi_{1}(\omega) \bar{\phi}_{2}(\omega) f(\omega) d \omega$. Corresponding to each element $\phi \in U_{\delta, \nu}$, there is a random variable $Z_{\phi}$ defined by identifying $e^{i \delta \omega^{\prime}(J+\nu)}$ with $Z(\delta(J+\nu))$ and extending the correspondence first to finite linear combinations of these elements and then to limits of such linear combinations with respect to the norm on $U_{\delta, \nu}$. We then have $\operatorname{var}\left(\int v(x) Z(x) d x-Z_{\delta}\right)=\int f(\omega) \mid V(\omega)-$ $\left.\phi(\omega)\right|^{2} d \omega$. The Hilbert space $U_{\delta}=U_{\delta, 0}$ is equivalent to the space of functions $U(\omega)=a(\omega)+i b(\omega)$ with $a$ and $b$ real, $a$ even, $b$ odd and both with period $2 \pi \delta^{-1}$ in each of its arguments with $\int f(\omega)|U(\omega)|^{2} d \omega<\infty$, as long as we equate functions $g_{0}(\omega)$ and $g_{1}(\omega)$ that satisfy $\int f(\omega)\left|g_{0}(\omega)-g_{1}(\omega)\right|^{2} d \omega=0$. Furthermore, $\phi(\omega) \in U_{\delta, \nu}$ if and only if $e^{-i \delta \omega^{\prime} v} \phi(\omega) \in U_{\delta}$. In particular, the function $\phi_{0}$ which equals $V$ on $A\left(\delta^{-1}\right)$ and for which $e^{-i \delta \omega^{\prime} \nu} \phi_{0}(\omega)$ has period $2 \pi \delta^{-1}$ in each argument is in $U_{\delta, \nu}$. Then

$$
\begin{aligned}
& \operatorname{var}\left(\int v(x) Z(x) d x-Z_{\phi_{0}}\right) \\
& \quad=\int_{A\left(\delta^{-1}\right)} \sum^{\prime} f\left(\omega+2 \pi \delta^{-1} J\right)\left|V\left(\omega+2 \pi \delta^{-1} J\right)-e^{-i 2 \pi J^{\prime} \nu} V(\omega)\right|^{2} d \omega \\
& \quad=S\left(\delta^{-1}\right)+R_{\delta},
\end{aligned}
$$

where

$$
\begin{aligned}
\left|R_{\delta}\right| \leq & \int_{\mathbb{R}^{d} \backslash A\left(\delta^{-1}\right)} f(\omega)|V(\omega)|^{2} d \omega \\
& +2\left\{\int_{\mathbb{R}^{d} \backslash A\left(\delta^{-1}\right)} f(\omega)|V(\omega)|^{2} d \omega \sum^{\prime} \int_{A\left(\delta^{-1}\right)} f\left(\omega+2 \pi \delta^{-1} J\right)|V(\omega)|^{2} d \omega\right\}^{1 / 2} .
\end{aligned}
$$

Under (2.1), we have $S\left(\delta^{-1}\right) \asymp \gamma\left(\delta^{-1}\right)$ and

$$
\int_{\mathbb{R}^{d} \backslash A\left(\delta^{-1}\right)} f(\omega)|V(\omega)|^{2} d \omega=O\left(\gamma\left(\delta^{-1}\right) \int_{\mathbb{R}^{d} \backslash A\left(\delta^{-1}\right)}|V(\omega)|^{2} d \omega\right)=o\left(\gamma\left(\delta^{-1}\right)\right),
$$

since $\int v(x)^{2} d x<\infty$ implies $\int|V(\omega)|^{2} d \omega<\infty$. It follows that $\left|R_{\delta}\right|=o\left(\gamma\left(\delta^{-1}\right)\right)$ and that the suboptimal predictor defined by $Z_{\phi_{0}}$ has asymptotic meansquared error $S\left(\delta^{-1}\right)$. 
Next we need to show that the optimal predictor does no better asymptotically than given by Proposition 2.1. Following Hannan [(1970), page 167], it is easy to show that the optimal predictor is given by the random variable corresponding to

$$
h_{\delta}(\omega)=\frac{\sum f\left(\omega+2 \pi \delta^{-1} J\right) V\left(\omega+2 \pi \delta^{-1} J\right) e^{-i 2 \pi J^{\prime} \nu}}{\sum f\left(\omega+2 \pi \delta^{-1} J\right)} .
$$

Then

$$
\begin{gathered}
\operatorname{var}\left(\int v(x)\left\{Z(x)-\hat{Z}_{\delta}(x)\right\} d x\right) \\
\quad \geq \int_{A\left(\delta^{-1}\right)} \sum^{\prime} f\left(\omega+2 \pi \delta^{-1} J\right)\left|V\left(\omega+2 \pi \delta^{-1} J\right)-e^{i 2 \pi J^{\prime} \nu} h_{\delta}(\omega)\right|^{2} d \omega \\
\quad \geq \int_{A\left(\delta^{-1}\right)} \sum^{\prime} f\left(\omega+2 \pi \delta^{-1} J\right)\left\{|V(\omega)|-\left|V(\omega)-h_{\delta}(\omega)\right|\right. \\
\left.-\left|V\left(\omega+2 \pi \delta^{-1} J\right)\right|\right\}^{2} d \omega .
\end{gathered}
$$

Proposition 2.1 will follow if we can show $\operatorname{var}\left(\int v(x)\left\{Z(x)-\hat{Z}_{\delta}(x)\right\} d x\right) \geq$ $S\left(\delta^{-1}\right)+o\left(\gamma\left(\delta^{-1}\right)\right)$. Since

$$
\begin{aligned}
& \left\{|V(\omega)|-\left|V(\omega)-h_{\delta}(\omega)\right|-\left|V\left(\omega+2 \pi \delta^{-1} J\right)\right|\right\}^{2} \\
& \quad \geq|V(\omega)|^{2}-2|V(\omega)|\left\{\left|V(\omega)-h_{\delta}(\omega)\right|+\left|V\left(\omega+2 \pi \delta^{-1} J\right)\right|\right\},
\end{aligned}
$$

from (2.2), it suffices to show

$$
\begin{aligned}
& \int_{A\left(\delta^{-1}\right)} \sum^{\prime} f\left(\omega+2 \pi \delta^{-1} J\right)|V(\omega)| \\
& \quad \times\left\{\left|V(\omega)-h_{\delta}(\omega)\right|+\left|V\left(\omega+2 \pi \delta^{-1} J\right)\right|\right\} d \omega=o\left(\gamma\left(\delta^{-1}\right)\right) .
\end{aligned}
$$

By (2.1), $f\left(\omega+2 \pi \delta^{-1} J\right) / \gamma\left(\delta^{-1}|J|\right)$ is uniformly bounded by some constant $C$ for all $\omega \in A\left(\delta^{-1}\right), J \neq 0$ and all $\delta$ sufficiently small, so

$$
\begin{aligned}
\int_{A\left(\delta^{-1}\right)} \sum^{\prime} f\left(\omega+2 \pi \delta^{-1} J\right)|V(\omega)|\left\{\left|V(\omega)-h_{\delta}(\omega)\right|+\left|V\left(\omega+2 \pi \delta^{-1} J\right)\right|\right\} d \omega \\
\leq C \sum^{\prime} \gamma\left(\delta^{-1}|J|\right) \int_{A\left(\delta^{-1}\right)}|V(\omega)|\left\{\left|V(\omega)-h_{\delta}(\omega)\right|+\left|V\left(\omega+2 \pi \delta^{-1} J\right)\right|\right\} d \omega \\
\leq 2 C\left\{\int|V(\omega)|^{2} d \omega\right\}^{1 / 2} \sum^{\prime} \gamma\left(\delta^{-1}|J|\right)\left[\left\{\int_{A\left(\delta^{-1}\right)}\left|V(\omega)-h_{\delta}(\omega)\right|^{2} d \omega\right\}^{1 / 2}\right. \\
\left.+\left\{\int_{A\left(\delta^{-1}\right)}\left|V\left(\omega+2 \pi \delta^{-1} J\right)\right|^{2} d \omega\right\}^{1 / 2}\right] .
\end{aligned}
$$


Thus, (2.3) easily follows if $\int_{A\left(\delta^{-1}\right)}\left|V(\omega)-h_{\delta}(\omega)\right|^{2} d \omega=o(1)$. We have

$$
\begin{aligned}
\int_{A\left(\delta^{-1}\right)}\left|V(\omega)-h_{\delta}(\omega)\right|^{2} d \omega \\
\leq 2 \int_{A\left(\delta^{-1}\right)}|V(\omega)|^{2}\left\{\frac{\sum^{\prime} f\left(\omega+2 \pi \delta^{-1} J\right)}{\sum f\left(\omega+2 \pi \delta^{-1} J\right)}\right\}^{2} d \omega \\
\quad+2 \int_{A\left(\delta^{-1}\right)}\left\{\frac{\sum^{\prime} f\left(\omega+2 \pi \delta^{-1} J\right)\left|V\left(\omega+2 \pi \delta^{-1} J\right)\right|}{\sum f\left(\omega+2 \pi \delta^{-1} J\right)}\right\}^{2} d \omega .
\end{aligned}
$$

The first integral on the right-hand side of (2.4) tends to 0 as $\delta \downarrow 0$ by dominated convergence since the integrand tends to 0 and is dominated by $|V(\omega)|^{2}$, which is integrable over $\mathbb{R}^{d}$. The second term on the right-hand side of (2.4) is bounded by

$$
2 \int_{A\left(\delta^{-1}\right)} \frac{\Sigma^{\prime} f\left(\omega+2 \pi \delta^{-1} J\right)^{2} \Sigma^{\prime}\left|V\left(\omega+2 \pi \delta^{-1} J\right)\right|^{2}}{\left\{\Sigma f\left(\omega+2 \pi \delta^{-1} J\right)\right\}^{2}} d \omega .
$$

Using (2.1), it follows that $\Sigma^{\prime} f\left(\omega+2 \pi \delta^{-1} J\right)^{2} /\left\{\sum f\left(\omega+2 \pi \delta^{-1} J\right)\right\}^{2}$ is uniformly bounded for $\omega \in A\left(\delta^{-1}\right)$ and all $\delta$ sufficiently small, so the second term on the right-hand side of (2.4) tends to 0 as $\delta \downarrow 0$. Thus, (2.3) and Proposition 2.1 follow.

Note that the condition $f(\omega)>0$ almost everywhere is necessary. Let $B$ be the set on which $f$ is 0 , let $1\{\cdot\}$ be an indicator function and define $\phi(\omega)=$ $V(\omega) 1\{\omega \in B\}$ on $A\left(\delta^{-1}\right)$, and elsewhere by the periodicity of $e^{-i \delta \omega^{\prime} \nu} \phi(\omega)$, so that $\phi \in U_{\delta, \nu}$. For $f(\omega)=0$ on a set of positive measure, the predictor $Z_{\phi}$ has asymptotic mean-squared error $S\left(\delta^{-1}\right)-\int_{B} \Sigma^{\prime} f\left(\omega+2 \pi \delta^{-1} J\right)|V(\omega)|^{2} d \omega$, which is nonnegligibly smaller than $S\left(\delta^{-1}\right)$ as long as $\int_{B}|V(\omega)|^{2} d \omega>0$.

3. Integrals over the unit cube. In practice, we normally only observe $Z$ over a finite lattice and $v$ has bounded support. As an important special case, we will consider predicting $\int_{[0,1]^{d}} v(x) Z(x) d x$ based on observing $Z\left(m^{-1}(J-h)\right)$, where $m$ is a positive integer, $h=\frac{1}{2}(1, \ldots, 1)$ and $J$ ranges over the $m^{d}$ points in $L(m)=\{1, \ldots, m\}^{d}$. Stein (1993) considers this problem when $v(x) \equiv 1$. This section considers the asymptotic optimality of the natural predictor

$$
\hat{Z}_{m}=m^{-d} \sum_{L(m)} v\left(\frac{J-h}{m}\right) Z\left(\frac{J-h}{m}\right)
$$

for $\int_{[0,1]^{d}} v(x) Z(x) d x$.

Proposition 3.1. If $f$ is bounded and satisfies (2.1), $\gamma$ is regularly varying with exponent $-p$ with $p<4$ and $v$ has bounded partial derivatives of order $d+1$ on $[0,1]^{d}$, then, as $m \rightarrow \infty, \operatorname{var}\left(\int_{[0,1]^{d}} v(x) Z(x) d x-\hat{Z}_{m}\right) \sim S(m)$. 
Since we require $p>d$ for $f$ to be integrable, this proposition is vacuous for $d \geq 4$.

Proof of Proposition 3.1. The key to proving this result is the following lemma bounding the difference between a Fourier transform of a smooth function with support on the unit cube and its approximation by a finite sum, proven in the Appendix.

LEMMA 3.1. Suppose $v$ has bounded partial derivatives of order $d+1$ on $[0,1]^{d}$. Then there exists a constant $C$ independent of $m$ and $\omega$ such that

$$
\begin{gathered}
\left|\int_{[0,1]^{d}} v(x) e^{i \omega^{\prime} x} d x-m^{-d} \sum_{L(m)} v\left(\frac{J-h}{m}\right) e^{i \omega^{\prime}(J-h) / m}\right| \\
\leq \frac{C}{m^{2}}\left\{1+\frac{(\log m)^{2 d}\left(1+|\omega|^{2}\right)}{\prod_{j=1}^{d}\left(1+\left|\omega_{j}\right|\right)}\right\}
\end{gathered}
$$

for all $\omega \in A(m)$, where $\omega_{j}$ is the jth component of $\omega$.

To apply Lemma 3.1 to Proposition 3.1, write

$$
\begin{aligned}
& \operatorname{var}\left(\int_{[0,1]^{d}} v(x) Z(x) d x-\hat{Z}_{m}\right) \\
& =\int_{A(m)} f(\omega)\left|V(\omega)-m^{-d} \sum_{L(m)} v\left(\frac{J-h}{m}\right) e^{i \omega^{\prime}(J-h) / m}\right|^{2} d \omega \\
& \quad+\int_{A(m)} \sum_{K}^{\prime} f(\omega+2 \pi m K) \mid V(\omega+2 \pi m K) \\
& -\left.m^{-d} \sum_{J \in L(m)} v\left(\frac{J-h}{m}\right) e^{i\left\{\omega^{\prime}(J-h) / m-2 \pi K^{\prime} h\right\}}\right|^{2} d \omega .
\end{aligned}
$$

The second term on the right-hand side of (3.1) is bounded by

$$
\begin{aligned}
\int_{A(m)} & \sum_{K}^{\prime} f(\omega+2 \pi m K)\left[|V(\omega)|^{2}+2|V(\omega)|\right. \\
& \times\left\{|V(\omega+2 \pi m K)|+\left|V(\omega)-m^{-d} \sum_{J \in L(m)} v\left(\frac{J-h}{m}\right) e^{i \omega^{\prime}(J-h) / m}\right|\right\} \\
& \left.+2|V(\omega+2 \pi m K)|^{2}+\left.2\left|V(\omega)-m^{-d} \sum_{J \in L(m)} v\left(\frac{J-h}{m}\right) e^{i \omega^{\prime}(J-h) / m}\right|\right|^{2}\right] d \omega
\end{aligned}
$$


which we want to show is $S(m)+o(\gamma(m))$. Similar to the proof of (2.3), this result will follow if

$$
\int_{A(m)}\left|V(\omega)-m^{-d} \sum_{L(m)} v\left(\frac{J-h}{m}\right) e^{i \omega^{\prime}(J-h) / m}\right|^{2} d \omega=o(1),
$$

which follows from Lemma 3.1 and $\int_{A(m)}\left(1+|\omega|^{2}\right)^{2} / \prod_{j=1}^{d}\left(1+\left|\omega_{j}\right|\right)^{2} d \omega=$ $O\left(\mathrm{~m}^{3}\right)$. Then Proposition 3.1 obtains if we can show the first term on the right-hand side of (3.1) is $o(\gamma(m))$. By (2.1) and $f$ bounded, we have that, for any $q<p, f(\omega)=O\left(\left(1+|\omega|^{q}\right)^{-1}\right)$ and

$$
\frac{\left(1+|\omega|^{2}\right)^{2}}{\left(1+|\omega|^{q}\right) \prod_{j=1}^{d}\left(1+\left|\omega_{j}\right|\right)^{2}}=O\left(\frac{1+\sum_{j=1}^{d} \omega_{j}^{4-q}}{\prod_{j=1}^{d}\left(1+\left|\omega_{j}\right|\right)^{2}}\right),
$$

so, by Lemma 3.1,

$$
\begin{aligned}
\int_{A(m)} & f(\omega)\left|V(\omega)-m^{-d} \sum_{L(m)} v\left(\frac{J-h}{m}\right) e^{i \omega^{\prime}(J-h) / m}\right|^{2} d \omega \\
= & O\left(\frac{(\log m)^{4 d}}{m^{4}} \int_{A(m)} \frac{1+\sum_{j=1}^{d} \omega_{j}^{4-q}}{\prod_{j=1}^{d}\left(1+\left|\omega_{j}\right|\right)^{2}} d \omega\right) \\
= & O\left((\log m)^{4 d}\left(m^{-4}+m^{-q-1}\right)\right) .
\end{aligned}
$$

Since $\gamma(m) m^{r} \rightarrow \infty$ for any $r>p$, by choosing $q=p-\frac{1}{2}$, say, we get that the first term on the right-hand side of (3.1) is $o(\gamma(m))$ for $p<4$.

Propositions 2.1 and 3.1 imply that if the conditions on $f$ and $v$ in Proposition 3.1 are met and $f$ is positive almost everywhere, then $\hat{Z}_{m}$ does as well asymptotically as the optimal predictor based on the infinite lattice of observations at $(J-h) / m$ and thus does as well asymptotically as the best predictor based on observations at $(J-h) / m$ for $J \in L(m)$.

4. Extension to smoother processes. This section describes a class of predictors for which the results of Section 3 apply to values for the exponent $p$ that are greater than or equal to 4 . The problem with the predictor in Section 3 is that it essentially predicts the integral of $Z$ over the cube of side $m^{-1}$ centered at $(J-h) / m$ by $m^{-d} Z((J-h) / m)$. This natural predictor is not good enough for smooth processes. In spectral terms, this corresponds to approximating

$$
\eta_{m J}(\omega)=\int_{[-1 /(2 m), 1 /(2 m)]^{d}} v\left(\frac{J-h}{m}+x\right) e^{i \omega^{\prime} x} d x
$$

by $m^{-d} v((J-h) / m) \exp \left(i \omega^{\prime}(J-h) / m\right)$. To develop a better approximation, we first need some preliminary results. Define $h_{m}(s, n)=\int_{-1 /(2 m)}^{1 /(2 m)} e^{i s y} y^{n} d y$ and $\alpha_{m Q}(\omega)=\prod_{a=1}^{d} h_{m}\left(\omega_{a}, Q_{a}\right)$, where $Q=\left(Q_{1}, \ldots, Q_{d}\right)$. Define $D_{k}$ to be the 
subset of $\mathbb{Z}^{d}$ whose components are nonnegative and sum to $k$. Then, for $v$ possessing bounded partial derivatives through order $q$ on $[0,1]^{d}$,

$$
\eta_{m J}(\omega)=\sum_{k=0}^{q-1} \frac{1}{k !} \sum_{Q \in D_{k}} \alpha_{m Q}(\omega) v^{(Q)}\left(\frac{J-h}{m}\right)+O\left(m^{-d-q}\right),
$$

where the remainder is uniform in $\omega$ and $J \in L(m)$ and $v^{(Q)}(x)=$ $\left\{\partial^{Q_{1}+\cdots+Q_{d}} / \partial x_{1}^{Q_{1}} \cdots \partial x_{d}^{Q_{d}}\right\} v(x)$. Defining

$$
\beta_{j}(n)=1\{j+n \text { even }\} \frac{i^{j}}{j !(j+n+1) 2^{n+j}},
$$

we have

$$
h_{m}(s, n)=\frac{1}{m^{n+1}}\left\{\sum_{j=0}^{k} \beta_{j}(n)\left(\frac{s}{m}\right)^{j}+O\left(\left(\frac{s}{m}\right)^{k+1}\right)\right\},
$$

where the remainder is uniform for $|s / m| \leq \pi$.

We can now write a function of the form $\Sigma_{K} e^{i \omega^{\prime} K} \lambda_{K}$ that approximates $\eta_{m J}(\omega)$ well for $\omega \in A(m)$. For fixed nonnegative integers $r$ and $t_{0}, \ldots, t_{r}$, define

$$
\phi_{m}(\omega, J)=\sum_{b=0}^{r} \sum_{Q \in D_{b}} v^{(Q)}\left(\frac{J-h}{m}\right) \prod_{k=1}^{d}\left\{\frac{1}{m^{Q_{k}+1}} \sum_{l=0}^{t_{b}} \nu_{l t_{b}}\left(Q_{k}\right) e^{i \omega_{k} l / m}\right\},
$$

where $\nu_{l t}(n)$ for $l=0, \ldots, t$ are defined as the unique solution to the system of equations

$$
\beta_{j}(n)=\frac{i^{j}}{j !} \sum_{l=0}^{t} \nu_{l t}(n) l^{j} \quad \text { for } j=0, \ldots, t .
$$

We then have, for $|s / m| \leq \pi$,

$$
\left|h_{m}(s, n)-\frac{1}{m^{n+1}} \sum_{l=0}^{t} \nu_{l t}(n) e^{i s l / m}\right|=O\left(\frac{s^{t+1}}{m^{n+t+2}}\right),
$$

which implies

$$
\begin{gathered}
\left|\alpha_{m Q}(\omega)-\prod_{a=1}^{d}\left\{\frac{1}{m^{Q_{a}+1}} \sum_{l=0}^{t_{a}} \nu_{l t_{a}}\left(Q_{a}\right) e^{i s l / m}\right\}\right| \\
=O\left(\sum_{a=1}^{d} \frac{|\omega|^{t_{a}+1}}{m^{d+a+t_{a}+1}}\right) .
\end{gathered}
$$

One way to proceed would be to consider the predictor given by the random variable corresponding to $\Sigma_{L(m)} \phi_{m}(\omega, J) e^{i \omega^{\prime}(J-h) / m}$. For $p, v, r$ and $t_{0}, \ldots, t_{r}$ satisfying the conditions of Proposition 4.1 and $f$ the conditions of Proposition 2.1, this predictor is asymptotically optimal. However, it uses some observations outside $[0,1]^{d}$. Instead, defining $S_{m}(j)=1$ if $j \leq m-t_{M}$ and 
$S_{m}(j)=-1$ otherwise, where $t_{M}=\max \left(t_{0}, \ldots, t_{r}\right)$, let

$$
\psi_{m}(\omega, J)=\sum_{b=0}^{r} \sum_{Q \in D_{b}} v^{(Q)}\left(\frac{J-h}{m}\right) \prod_{k=1}^{d}\left\{\frac{1}{m^{Q_{k}+1}} \sum_{l=0}^{t_{b}} \nu_{l t_{b}}\left(Q_{k}\right) e^{i S_{m}\left(J_{k}\right) \omega_{k} l / m}\right\},
$$

where $J=\left(J_{1}, \ldots, J_{d}\right)$. Then the predictor corresponding to $\sum_{L(m)} \psi_{m}(\omega, J) e^{i \omega^{\prime}(J-h) / m}$, which we will call $\hat{Z}_{m}\left(r ; t_{0}, \ldots, t_{r}\right)$, is given by

$$
\begin{aligned}
\hat{Z}_{m}\left(r ; t_{0}, \ldots, t_{r}\right)= & \sum_{J \in L(m)} \sum_{b=0}^{r} \frac{1}{m^{b+d}} \sum_{Q \in D_{b}} v^{(Q)}\left(\frac{J-h}{m}\right) \\
& \times \sum_{l_{1}=0}^{t_{b}} \cdots \sum_{l_{d}=0}^{t_{b}}\left\{\prod_{k=1}^{d} \nu_{l_{k} t_{b}}\left(Q_{k}\right)\right\} \\
& \times Z\left(\frac{J-h+\left(S_{m}\left(J_{1}\right) l_{1}, \ldots, S_{m}\left(J_{d}\right) l_{d}\right)}{m}\right) .
\end{aligned}
$$

This predictor only uses observations in $[0,1]^{d}$.

Proposition 4.1. Suppose $f$ is bounded and satisfies (2.1) where $\gamma$ is regularly varying with exponent $-p, v$ has bounded partial derivatives of order $\max (r, d+2+\lfloor p / 2\rfloor)$ on $[0,1]^{d}$ and $r$ and $t_{0}, \ldots, t_{r}$ are nonnegative integers satisfying $r>p / 2$ and $t_{k}>p / 2-k+1$ for $k=0, \ldots, r$. Then, as $m \rightarrow \infty, \operatorname{var}\left(\int_{[0,1]^{d}} v(x) Z(x) d x-\hat{Z}_{m}\left(r ; t_{0}, \ldots, t_{r}\right)\right) \sim S(m)$.

Before proving this result, some comments are in order. The conditions on $v, r$ and $t_{0}, \ldots, t_{r}$ are stronger than necessary. In particular, Proposition 3.1 does not follow from Proposition 4.1. Furthermore, the definition of the predictor is somewhat arbitrary. For example, the predictor changes if $x_{j}$, the $j$ th component of $x$, is replaced by $1-x_{j}$. Thus, in practice, it may be desirable to use some other predictor that also possesses this optimality property. One possibility is to use the exactly optimal predictor under some spectral density $g$ for $Z$. I would conjecture that, for any $g$ satisfying (2.1), the resulting predictor would be asymptotically optimal if the true spectral density for $Z$ is given by $f$, where $f$ satisfies (2.1) and $g(\omega) / f(\omega)$ is bounded and does not tend to 0 as $|\omega| \rightarrow \infty$ at faster than an algebraic rate. Of course, for large $m$, it may not be feasible to calculate the exactly optimal predictor under $g$.

Schoenfelder (1982) studied the asymptotic behavior of predictors using random sampling locations. Among the sampling plans she considered, stratified random sampling had the fastest rates of convergence. Her conditions on the covariance function of $Z$ are neither more nor less general than (2.1) here. However, where they overlap, the rates of convergence given by Proposition 4.1 are always at least as fast as those given by Schoenfelder (1982) for stratified random sampling. If (2.1) holds, then her results give predictors based on stratified random sampling that have asymptotic mean-squared error of order $n^{-1-1 / d}$ for $f(\omega) \asymp|\omega|^{-d-1}$ and asymptotic mean-squared 
error of order $n^{-1-2 / d}$ for $f(\omega)=o\left(|\omega|^{-d-2}\right)$. Since $n=m^{d}$ here, the $n^{-1-1 / d}$ rate for $f(\omega) \asymp|\omega|^{-d-1}$ is the same as in Proposition 4.1. Furthermore, the $n^{-1-2 / d}$ rate for $f(\omega)=o\left(|\omega|^{-d-2}\right)$ is worse than that in Proposition 4.1, since $f(\omega)=o\left(|\omega|^{-d-2}\right)$ implies the asymptotic mean-squared error in Proposition 4.1 is $o\left(n^{-1-2 / d}\right)$.

Proof of Proposition 4.1. We have

$\operatorname{var}\left(\int_{[0,1]^{d}} v(x) Z(x) d x-\hat{Z}_{m}\left(r ; t_{0}, \ldots, t_{r}\right)\right)$

$$
\begin{aligned}
=\int_{A(m)} f(\omega)\left|V(\omega)-\sum_{L(m)} \psi_{m}(\omega, J) e^{i \omega^{\prime}(J-h) / m}\right|^{2} d \omega \\
+\int_{A(m)} \sum_{J}^{\prime} f(\omega+2 \pi m J) \mid V(\omega+2 \pi m J) \\
-\left.\sum_{K \in L(m)} \psi_{m}(\omega, K) e^{i \omega^{\prime}(K-h) / m-i 2 \pi J^{\prime} h}\right|^{2} d \omega
\end{aligned}
$$

From the definition of $\psi_{m}(\omega, K)$ and the conditions on $v$, we have $m^{d} \sum_{L(m)} e^{i \omega^{\prime}(J-h) / m} \psi_{m}(\omega, J)=\sum_{L(m)} \lambda_{m J} e^{i \omega^{\prime}(J-h) / m}$, where $\lambda_{m J}-v((J-$ $h) / m)=O\left(m^{-1}\right)$ for $J \in L\left(m-t_{M}\right)$ and $\lambda_{m J}=O(1)$ for $J \in L(m) \backslash L(m-$ $\left.t_{M}\right)$. Then, similar to the argument used to bound the second term on the right-hand side of (3.1), it can be shown that, as $m \rightarrow \infty$,

$$
\begin{aligned}
\int_{A(m)} \sum_{J}^{\prime} f(\omega+2 \pi m J) \mid & V(\omega+2 \pi m J) \\
& -\left.\sum_{K \in L(m)} \psi_{m}(\omega, K) e^{i \omega^{\prime}(K-h) / m-i 2 \pi J^{\prime} h}\right|^{2} d \omega \sim S(m) .
\end{aligned}
$$

Proposition 4.1 holds if we can show that the first term on the right-hand side of (4.3) is $o(\gamma(m))$. To prove this, first note that

$$
\begin{aligned}
\left|\int_{[0,1]^{d}} v(x) e^{i \omega^{\prime} x} d x-\sum_{L(m)} e^{i \omega^{\prime}(J-h) / m} \phi_{m}(\omega, J)\right|^{2} \\
=\left|\sum_{L(m)} e^{i \omega^{\prime}(J-h) / m}\left\{\int_{[-1 /(2 m), 1 /(2 m)]^{d}} v\left(x+\frac{J-h}{m}\right) e^{i \omega^{\prime} x} d x-\phi_{m}(\omega, J)\right\}\right|^{2} \\
=O\left(\sum_{b=0}^{L} \sum_{Q \in D_{b}} \mid \sum_{L(m)} e^{i \omega^{\prime}(J-h) / m} v^{(Q)}\left(\frac{J-h}{m}\right)\right. \\
\left.\quad \times\left.\left[\alpha_{m Q}(\omega)-\frac{1}{m^{d+b}} \prod_{k=1}^{d}\left\{\sum_{l=0}^{t_{b}} \nu_{l t_{b}}\left(Q_{k}\right) e^{i \omega_{k} l / m}\right\}\right]\right|^{2}+m^{-p-1}\right) \\
=O\left(\frac{(\log m)^{4 d}}{\prod_{k=1}^{d}\left(1+\omega_{k}^{2}\right)} \sum_{b=0}^{\lfloor p / 2\rfloor} \frac{|\omega|^{2 t_{b}+2}}{m^{2\left(b+1+t_{b}\right)}}+m^{-p-1}\right)
\end{aligned}
$$


using Lemma A.1 and (4.2). It follows that, for any fixed $\varepsilon>0$, using the conditions on $t_{0}, \ldots, t_{r}$,

$$
\int_{A(m)} f(\omega)\left|V(\omega)-\sum_{L(m)} e^{i \omega^{\prime}(J-h) / m} \phi_{m}(\omega, J)\right|^{2} d \omega=O\left(\frac{1}{m^{p+1-\varepsilon}}\right) .
$$

To finish the proof, it suffices to show

$$
\int_{A(m)} f(\omega)\left|\sum_{L(m)} e^{i \omega^{\prime} J / m}\left\{\psi_{m}(\omega, J)-\phi_{m}(\omega, J)\right\}\right|^{2} d \omega=o(\gamma(m)) .
$$

Now,

$\left|\sum_{L(m)} \exp \left\{i \omega^{\prime} J m^{-1}\right\}\left\{\psi_{m}(\omega, J)-\phi_{m}(\omega, J)\right\}\right|^{2}$

$$
\begin{gathered}
=O\left(\sum_{b=0}^{\lfloor p / 2\rfloor} \frac{1}{m^{2(d+b)}} \sum_{Q \in D_{b}} \mid \sum_{J \in L(m) \backslash L\left(m-t_{M}\right)} \exp \left\{i \omega^{\prime} J m^{-1}\right\} v^{(Q)}\left(\frac{J-h}{m}\right)\right. \\
\times \sum_{l_{1}, \ldots, l_{d}=0}^{t_{b}} \prod_{\gamma=1}^{d} \nu_{l_{\gamma} t_{b}}\left(Q_{\gamma}\right) \\
\left.\times\left.\left[\exp \left\{i \sum_{k=1}^{d} \omega_{k} l_{k} m^{-1}\right\}-\exp \left\{i \sum_{k=1}^{d} S_{m}\left(J_{k}\right) \omega_{k} l_{k} m^{-1}\right\}\right]\right|^{2}+m^{-p-1}\right) .
\end{gathered}
$$

Consider $J_{1}, \ldots, J_{c}$ fixed and $m-t_{M} \leq J_{k} \leq m$ for $k=1, \ldots, c$. For $\omega \in$ $A(m)$,

$$
\begin{aligned}
& \mid \sum_{J_{c+1, \ldots, J_{d}=1}}^{m} \exp \left\{i \omega^{\prime} J m^{-1}\right\} v^{(Q)}\left(\frac{J-h}{m}\right) \sum_{l_{1}, \ldots, l_{d}=0}^{t_{b}} \prod_{\gamma=1}^{d} \nu_{l_{\gamma} t_{b}}\left(Q_{\gamma}\right) \\
& \times\left[\exp \left\{i \sum_{k=1}^{d} \omega_{k} l_{k} m^{-1}\right\}-\exp \left\{i \sum_{k=1}^{d} S_{m}\left(J_{k}\right) \omega_{k} l_{k} m^{-1}\right\}\right] \mid \\
& =\mid \sum_{J_{c+1, \ldots, J_{d}=1}}^{m} \exp \left\{i \omega^{\prime} J m^{-1}\right\} v^{(Q)}\left(\frac{J-h}{m}\right) \sum_{l_{1}, \ldots, l_{c}=0}^{t_{b}} \prod_{\gamma=1}^{c} \nu_{l_{\gamma} t_{b}}\left(Q_{\gamma}\right) \\
& \times\left[\exp \left\{i \sum_{k=1}^{c} \omega_{k} l_{k} m^{-1}\right\}-\exp \left\{-i \sum_{k=1}^{c} \omega_{k} l_{k} m^{-1}\right\}\right] \\
& \times \prod_{k=c+1}^{d}\left\{\sum_{l=0}^{t_{b}} \nu_{l t_{b}}\left(Q_{k}\right) \exp \left\{i \omega_{k} l_{k} m^{-1}\right\}\right\} \mid \\
& =O\left(\frac{|\omega|^{t_{b}+1} m^{d-c-t_{b}-1}(\log m)^{2(d-c)}}{\prod_{k=c+1}^{d}\left(1+\left|\omega_{k}\right|\right)}\right),
\end{aligned}
$$


using Lemma 3.1 and

$$
\begin{aligned}
& \sum_{l_{1}, \ldots, l_{c}=0}^{t_{b}} \prod_{\gamma=1}^{c} \nu_{l_{\gamma} t_{b}}\left(Q_{\gamma}\right)\left[\exp \left\{i \sum_{k=1}^{c} \omega_{k} l_{k} m^{-1}\right\}-\exp \left\{-i \sum_{k=1}^{c} \omega_{k} l_{k} m^{-1}\right\}\right] \\
&=\prod_{a=1}^{c}\left\{\sum_{l=0}^{t_{b}} \nu_{l t_{b}}\left(Q_{a}\right) \exp \left\{i \omega_{a} l_{a} m^{-1}\right\}\right\}-\prod_{a=1}^{c}\left\{\sum_{l=0}^{t_{b}} \nu_{l t_{b}}\left(Q_{a}\right) \exp \left\{-i \omega_{a} l_{a} m^{-1}\right\}\right\} \\
&=\prod_{a=1}^{c}\left\{m^{Q_{a}+1} h_{m}\left(\omega_{a}, Q_{a}\right)+O\left(\left(\frac{\left|\omega_{a}\right|}{m}\right)^{t_{b}+1}\right)\right\} \\
& \quad-\prod_{a=1}^{c}\left\{m^{Q_{a}+1} h_{m}\left(-\omega_{a}, Q_{a}\right)+O\left(\left(\frac{\left|\omega_{a}\right|}{m}\right)^{t_{b}+1}\right)\right\} \\
&= O\left(\left(\frac{|\omega|}{m}\right)^{t_{b}+1}\right),
\end{aligned}
$$

where we have used the definitions of $\nu_{l t}(n)$ and $h_{m}(s, n)$ and the fact that $h_{m}(s, n)$ is an even function of $s$. Since there are only a fixed finite number of terms for which $m-t_{M} \leq J_{k} \leq m$ for $k=1, \ldots, c$, it follows from (4.5) and (4.6) that

$$
\begin{aligned}
& \left|\sum_{L(m)} e^{i \omega^{\prime} J / m}\left\{\psi_{m}(\omega, J)-\phi_{m}(\omega, J)\right\}\right|^{2} \\
& \quad=O\left(\sum_{b=0}^{L p / 2\rfloor} \frac{(\log m)^{4 d}|\omega|^{2 t_{b}+2}}{m^{2\left(b+t_{b}+1\right)}} \sum_{c=1}^{d} \frac{m^{-2 c}}{\prod_{l=c+1}^{d}\left(1+\omega_{k}^{2}\right)}+m^{-p-1}\right) .
\end{aligned}
$$

Then (4.4) and hence Proposition 4.1 follow from the conditions on $t_{0}, \ldots, t_{r}$ and the bound $\int_{A(m)} f(\omega)|\omega|^{2 t_{b}+2} / \prod_{k=c+1}^{d}\left(1+\omega_{k}^{2}\right) d \omega=O\left(1+m^{2 t_{b}+2+c-p+\varepsilon}\right)$ for any $\varepsilon>0$.

\section{APPENDIX}

Proof of Lemma 3.1. Let $A$ be an upper bound on $v$ and all of its partial derivatives through order $d+1$ on $[0,1]^{d}$. Define $v_{j}(x)=\left(\partial / \partial x_{j}\right) v(x)$. Then

$$
\begin{aligned}
& \left|\int_{[0,1]^{d}} v(x) e^{i \omega^{\prime} x} d x-m^{-d} \sum_{L(m)} v\left(\frac{J-h}{m}\right) e^{i \omega^{\prime}(J-h) / m}\right| \\
& \leq\left|\sum_{L(m)} \int_{[-1 /(2 m), 1 /(2 m)]^{d}} e^{i \omega^{\prime}\{x+(J-h) / m\}} \sum_{j=1}^{d} x_{j} v_{j}\left(\frac{J-h}{m}\right) d x\right|+\frac{A d^{2}}{m^{2}} \\
& \quad+\left|\sum_{L(m)} v\left(\frac{J-h}{m}\right) e^{i \omega^{\prime}(J-h) / m}\left\{\int_{[-1 /(2 m), 1 /(2 m)]^{d}} e^{i \omega^{\prime} x} d x-m^{-d}\right\}\right|
\end{aligned}
$$




$$
\begin{aligned}
&=\mid \sum_{L(m)} e^{i \omega^{\prime}(J-h) / m} \sum_{j=1}^{d} {\left[v_{j}\left(\frac{J-h}{m}\right)\left\{\frac{1}{i m \omega_{j}} \cos \left(\frac{\omega_{j}}{2 m}\right)-\frac{2}{i \omega_{j}^{2}} \sin \left(\frac{\omega_{j}}{2 m}\right)\right\}\right.} \\
&\left.\times \prod_{k \neq j} \frac{2}{\omega_{k}} \sin \left(\frac{\omega_{k}}{2 m}\right)\right] \mid+\frac{A d^{2}}{m^{2}} \\
&+\left|\sum_{L(m)} v\left(\frac{J-h}{m}\right) e^{i \omega^{\prime}(J-h) / m}\right|\left|\prod_{j=1}^{d} \frac{2}{\omega_{j}} \sin \left(\frac{\omega_{j}}{2 m}\right)-m^{-d}\right| \\
& \leq \sum_{j=1}^{d}\left|\sum_{L(m)} v_{j}\left(\frac{J-h}{m}\right) e^{i \omega^{\prime}(J-h) / m}\right| \frac{\left|\omega_{j}\right|}{m^{d+2}} \\
&+\frac{A d^{2}}{m^{2}}+\left|\sum_{L(m)} v\left(\frac{J-h}{m}\right) e^{i \omega^{\prime}(J-h) / m}\right| \frac{|\omega|^{2}}{m^{d+2}} .
\end{aligned}
$$

Lemma 3.1 holds by applying the following bound to $v(x)$ and $v_{j}(x)$ for $j=1, \ldots, d$.

LEMMA A.1. If $u: \mathbb{R}^{d} \rightarrow \mathbb{R}$ has bounded partial derivatives of order $d$ on $[0,1]^{d}$, then for all $\omega \in A(m)$ there exists a constant $B$ depending on $u$ such that

$$
\left|\sum_{L(m)} u\left(\frac{J}{m}\right) e^{i \omega^{\prime} J / m}\right| \leq \frac{B m^{d}(\log m)^{2 d}}{\prod_{j=1}^{d}\left(1+\left|\omega_{j}\right|\right)}
$$

Proof. Suppose $2^{n} \leq m<2^{n+1}$. For nonnegative integers $n_{1}, \ldots, n_{d}$ that are at most $n$, consider, for some real-valued function $t$ on $\mathbb{R}^{d}$,

$$
\sum_{J_{1}=0}^{2^{n_{1}}-1} \cdots \sum_{J_{d}=0}^{2^{n_{d}}-1} t\left(\frac{J}{m}\right) e^{i \omega^{\prime} J / m},
$$

where $J=\left(J_{1}, \ldots, J_{d}\right)$. We can write $J_{k}=\sum_{l=1}^{n_{k}} j_{k l} 2^{n_{k}-l}$, where each $j_{k l}$ is 0 or 1 and we set $J_{k}=0$ if $n_{k}=0$. Then

$$
\begin{aligned}
t\left(\frac{J}{m}\right)= & \sum_{\alpha_{1}=0}^{n_{1}} \cdots \sum_{\alpha_{d}=0}^{n_{d}} \sum_{\beta_{1}=0}^{1} \cdots \sum_{\beta_{d}=0}^{1}(-1)^{\sum_{r=1}^{d} \beta_{r}} \\
& \times t\left(\sum_{l=1}^{\alpha_{1}-\beta_{1}} j_{1 l} 2^{n_{1}-l} m^{-1}, \ldots, \sum_{l=1}^{\alpha_{d}-\beta_{d}} j_{d l} 2^{n_{d}-l} m^{-1}\right)
\end{aligned}
$$

where $\sum_{l=1}^{0}$ is defined to be 0 and $t\left(\sum_{l=1}^{b_{1}} j_{1 l} 2^{n_{1}-l} m^{-1}, \ldots, \sum_{l=1}^{b_{d}} j_{d l} 2^{n_{d}-l} m^{-1}\right)$ 
$=0$ if any $b_{k}=-1$. Plugging this expression into (A.1) yields

$$
\begin{aligned}
\sum_{J_{1}=0}^{2^{n_{1}}-1} & \cdots \sum_{J_{d}=0}^{2^{n_{d}}-1} t\left(\frac{J}{m}\right) \exp \left\{i \omega^{\prime} J m^{-1}\right\} \\
= & \sum_{j_{11}=0}^{1} \exp \left\{i \omega_{1} j_{11} 2^{n_{1}-1} m^{-1}\right\} \cdots \sum_{j_{1 n_{1}=0}}^{1} \exp \left\{i \omega_{1} j_{1 n_{1}} m^{-1}\right\} \\
& \cdots \sum_{j_{d 1}=0}^{1} \exp \left\{i \omega_{d} j_{d 1} 2^{n_{d}-1} m^{-1}\right\} \\
& \cdots \sum_{j_{d n_{d}}=0}^{1} \exp \left\{i \omega_{d} j_{d n_{d}} m^{-1}\right\} \sum_{\alpha_{1}=0}^{n_{1}} \cdots \sum_{\alpha_{d}=0}^{n_{d}} \sum_{\beta_{1}=0}^{1} \cdots \sum_{\beta_{d}=0}^{1}(-1)^{\sum_{r=1}^{d} \beta_{r}} \\
& \times t\left(\sum_{l=1}^{\alpha_{1}-\beta_{1}} j_{1 l} 2^{n_{1}-l} m^{-1}, \ldots, \sum_{l=1}^{\alpha_{d}-\beta_{d}} j_{d l} 2^{n_{d}-l} m^{-1}\right)
\end{aligned}
$$

(A.2)

$$
\begin{aligned}
= & \sum_{\alpha_{1}=0}^{n_{1}} \cdots \sum_{\alpha_{d}=0}^{n_{d}} \sum_{j_{11}=0}^{1} \exp \left\{i \omega_{1} j_{11} 2^{n_{1}-1} m^{-1}\right\} \\
& \cdots \sum_{j_{1 \alpha_{1}}=0}^{1} \exp \left\{i \omega_{1} j_{1 \alpha_{1}} 2^{n_{1}-\alpha_{1}} m^{-1}\right\} \\
& \cdots \sum_{j_{d 1}=0}^{1} \exp \left\{i \omega_{d} j_{d 1} 2^{n_{d}-1} m^{-1}\right\} \cdots \sum_{j_{d \alpha_{d}}=0}^{1} \exp \left\{i \omega_{d} j_{d \alpha_{d}} 2^{n_{d}-\alpha_{d}} m^{-1}\right\} \\
& \times \sum_{\beta_{1}=0}^{1} \cdots \sum_{\beta_{d}=0}^{1}(-1)^{\sum_{r=1}^{d} \beta_{r}} t\left(\sum_{l=1}^{\alpha_{1}-\beta_{1}} j_{1 l} 2^{n_{1}-l} m^{-1}, \ldots, \sum_{l=1}^{\alpha_{d}-\beta_{d}} j_{d l} 2^{n_{d}-l} m^{-1}\right) \\
& \times \prod_{q=1}^{d} \prod_{k=\alpha_{q}+1}^{n_{q}}\left[1+\exp \left\{i \omega_{q} 2^{n_{q}-k} m^{-1}\right\}\right],
\end{aligned}
$$

where the term

$$
\sum_{j_{k 1}=0}^{1} \exp \left\{i \omega_{k} j_{k 1} 2^{n_{k}-1} m^{-1}\right\} \cdots \sum_{j_{k \alpha_{k}}=0}^{1} \exp \left\{i \omega_{k} j_{k \alpha_{k}} 2^{n_{k}-\alpha_{k}} m^{-1}\right\}
$$

drops out if $\alpha_{k}=0$ and a product with no terms is set equal to 1 . The ability to write the discrete Fourier transform in this form is the basis for the fast Fourier transform. This same representation is also the key to proving Lemma A.1.

To bound (A.2), the first step is to show that, for any function $s: \mathbb{R}^{d} \rightarrow \mathbb{R}$ with bounded partial derivatives of order $d$ on $[0,1]^{d}$, there exists a constant 
$C$ such that, for all $\varepsilon_{1}, \ldots, \varepsilon_{d} \in[0,1]$,

$$
\left|\sum_{\beta_{1}=0}^{1} \cdots \sum_{\beta_{d}=0}^{1}(-1)^{\sum_{r=1}^{d} \beta_{r}} s\left(\beta_{1} \varepsilon_{1}, \ldots, \beta_{d} \varepsilon_{d}\right)\right| \leq C \prod_{j=1}^{d} \varepsilon_{j} .
$$

To prove (A.3), assume, without loss of generality, $\varepsilon_{d} \leq \cdots \leq \varepsilon_{1}$. Then (A.3) follows by noting

$$
\begin{aligned}
\sum_{\beta_{1}=0}^{1} \cdots & \sum_{\beta_{d}=0}^{1}(-1)^{\sum_{r=1}^{d} \beta_{r}} s\left(\beta_{1} \varepsilon_{1}, \ldots, \beta_{d} \varepsilon_{d}\right) \\
= & \sum_{\beta_{1}=0}^{1} \ldots \sum_{\beta_{d-1}=0}^{1}(-1)^{\sum_{r=1}^{d-1} \beta_{r}}\left\{s\left(\beta_{1} \varepsilon_{1}, \ldots, \beta_{d-1} \varepsilon_{d-1}, \varepsilon_{d}\right)\right. \\
& \left.-s\left(\beta_{1} \varepsilon_{1}, \ldots, \beta_{d-1} \varepsilon_{d-1}, 0\right)\right\} \\
= & \sum_{\beta_{1}=0}^{1} \ldots \sum_{\beta_{d-1}=0}^{1}(-1)^{\sum_{r=1}^{d-1} \beta_{r}} \sum_{k=1}^{d-1} \frac{\varepsilon_{d}^{k}}{k !} \\
& \times s^{(0, \ldots, 0, k)}\left(\beta_{1} \varepsilon_{1}, \ldots, \beta_{d-1} \varepsilon_{d-1}, 0\right)+C_{1} \varepsilon_{d}^{d} \\
= & \sum_{\beta_{1}=0}^{1} \ldots \sum_{\beta_{d-2}=0}^{1}(-1)^{\sum_{r=1}^{d-2} \beta_{r}} \sum_{k=1}^{d-1} \frac{\varepsilon_{d}^{k}}{k !} \sum_{l=1}^{d-k-1} \frac{\varepsilon_{d}^{l}-1}{l !} \\
& \times s^{(0, \ldots, 0, l, k)}\left(\beta_{1} \varepsilon_{1}, \ldots, \beta_{d-2} \varepsilon_{d-2}, 0,0\right)+C_{2} \varepsilon_{d-1}^{d-1} \varepsilon_{d} \\
= & \ldots \quad C_{d} \prod_{j=1}^{d} \varepsilon_{j},
\end{aligned}
$$

where the $C_{j}$ 's are bounded by constants independent of the $\varepsilon_{j}$ 's. In fact, if $D$ bounds the partial derivatives through order $d$ of $s$ on $[0,1]^{d}$, then it can be shown that we can take $C=(2 d)^{d} D / d$ ! in (A.3).

If $t$ and all of its partial derivatives through order $d$ on $[0,1]^{d}$ are bounded by $D$, then applying (A.3) and the identity $\left|\Pi_{k=1}^{r}\left(1-e^{i \lambda 2^{-k}}\right)\right|=$ $\left|\sin (\lambda / 2) / \sin \left(\lambda / 2^{r+1}\right)\right|$ to (A.2) yields

$$
\begin{aligned}
\left|\sum_{J_{1}=0}^{2^{n_{1}}-1} \cdots \sum_{J_{d}=0}^{2^{n_{d}}-1} t\left(\frac{J}{m}\right) e^{i \omega^{\prime} J / m}\right| \\
\leq \sum_{\alpha_{1}=0}^{n_{1}} \cdots \sum_{\alpha_{d}=0}^{n_{d}} 2^{\alpha_{1}+\cdots+\alpha_{d}} \frac{(2 d)^{d} D}{d !} \prod_{\substack{j=1 \\
\alpha_{j}>0}}^{d} 2^{n_{j}-\alpha_{j}} \\
\quad \times m^{-1} \prod_{j=1}^{d}\left|\frac{\sin \left(\omega_{j} 2^{n_{j}-\alpha_{j}-1} m^{-1}\right)}{\sin \left\{\omega_{j} /(2 m)\right\}}\right|
\end{aligned}
$$


Now, $2^{n_{j}} \leq 2^{n} \leq m$, so

$$
2^{\alpha_{1}+\cdots+\alpha_{d}} \prod_{\substack{j=1, \alpha_{j}>0}}^{d} 2^{n_{j}-\alpha_{j}} m^{-1} \leq 1
$$

and, for $\omega \in A(m)$,

$$
\left|\frac{\sin \left(\omega_{j} 2^{n_{j}-\alpha_{j}-1} m^{-1}\right)}{\sin \left\{\omega_{j} /(2 m)\right\}}\right| \leq \frac{\min \left(1,\left|\omega_{j}\right|\right)}{\left|\omega_{j}\right| /(2 \pi m)} \leq \frac{4 \pi m}{1+\left|\omega_{j}\right|},
$$

using $\min (x, y) \leq 2 x y /(x+y)$ for $x, y$ positive. Thus,

$$
\begin{aligned}
& \left|\sum_{J_{1}=0}^{2^{n_{1}}-1} \cdots \sum_{J_{d}=0}^{2^{n_{d}}-1} t\left(\frac{J}{m}\right) e^{i \omega^{\prime} J / m}\right| \\
& \quad \leq \frac{(2 d)^{d} D}{d !}\{4 \pi m(n+1)\}^{d} \prod_{j=1}^{d}\left(1+\left|\omega_{j}\right|\right)^{-1}
\end{aligned}
$$

for $\omega \in A(m)$. Finally, $\sum_{J} u(J / m) e^{i \omega^{\prime} J / m}$ can be written as a sum of at most $(n+1)^{d}$ sums like

$$
\sum_{J_{1}=0}^{2^{n_{1}}-1} \cdots \sum_{J_{d}=0}^{2^{n_{d}}-1} t\left(\frac{J}{m}\right) e^{i \omega^{\prime} J / m},
$$

where $t(x)$ will be of the form $u\left(x+\left(1-2^{-n_{1}}, \cdots, 1-2^{-n_{d}}\right)\right)$, so Lemma A.1 follows from (A.4).

\section{REFERENCES}

Cressie, N. A. C. (1993). Statistics for Spatial Data, rev. ed. Wiley, New York.

Feller, W. (1971). An Introduction to Probability Theory and Its Applications 2, 2nd ed. Wiley, New York.

Hannan, E. J. (1970). Multiple Time Series. Wiley, New York.

MAteRn, B. (1986). Spatial Variation, 2nd ed. Springer, Berlin.

RIPLEY, B. D. (1981). Spatial Statistics. Wiley, New York.

SchoENFELDER, C. (1978). Random designs for estimating integrals of stochastic processes. Mimeo Series 1201, Dept. Statistics, Univ. North Carolina.

SCHOENFELDER, C. (1982). Random designs for estimating integrals of stochastic processes: asymptotics. Center for Stochastic Processes Report 6, Dept. Statistics, Univ. North Carolina.

Schoenfelder, C. and CAmbanis, S. (1982). Random designs for estimating integrals of stochastic processes. Ann. Statist. $10526-538$.

Stein, M. L. (1993). Asymptotic properties of centered systematic sampling for predicting integrals of spatial processes. Ann. Appl. Probab. 3 874-880.

Tubilla, A. (1975). Error convergence rates for estimates of multidimensional integrals of random functions. Technical Report 72, Dept. Statistics, Stanford Univ.

DEPARTMENT OF STATISTICS

UNIVERSITY OF CHICAGO

Chicago, Illinois 60637 